\title{
The Design and Implementation of Urban Noise Complaint System based on Mobile Crowd-sensing
}

\author{
Yonghui Jiang \\ School of Information Science and Technology, Hainan Normal University, Haikou, China \\ philjyh@163.com
}

Keywords: Mobile crowd-sensing; Urban noise; Complain system; Social task distribution

\begin{abstract}
As the difficulties to prove the existing urban noise, this paper designs and implements a urban noise complaint system, which is based on mobile crowd-sensing. This system takes a social task distribution mechanism, so that more and more users can use the phone as a mobile terminal to collect and measure the environmental noise data, and report the data to the server. Once the server summary the noise report information, it not only can provide the noise map to users and related department for inquiring, but also can remind the related department to deal with noise to reduce.
\end{abstract}

\section{Introduction}

With the process of urbanization in China, the problem of urban noise pollution has become increasingly prominent. The number of complaints about the noise pollution have been ranked the first in environmental pollution complaints.

The existing noise complaints are mainly for the public to call the related departments, and then the related departments to the noise scene for processing. However, the noise pollution is transient, localize and decentralized, the law-enforcing department is hard to get the noise evidence for punishment. So, it is meaningful to research a new noise complaint way.

With the development of mobile Internet technology, smart phones has been spread quickly. The smart phone has a variety of sensors, such as GPS, microphone, camera, gyroscope, accelerometer, etc. Through the mobile Internet of conscious or unconscious collaboration, the user can use the smart phone as a basic sensing unit to achieve the task of perception and distribution of data collect, and complete large-scale and complex social perception tasks. Therefore, this paper proposes a urban noise complaint system, which is based on mobile crowd-sensing. The user can use the smart phone to collect and measure the noise data and report it in time. To the server, the system generates the noise map for users and related departments to inquire, and then inform the related departments to deal with.

Compared with the existing environment noise monitoring system, this paper designs a social task distribution mechanism in the system, which aims to solve the problem of sparse data caused by the small number of users participating in the existing system. According to practice, the complaint system presented in this paper has solved the problem of difficulties in proofing evidence, and achieved better results.

\section{Related Work}

The crowd-sensing technology has been widely used in large-scale social perception tasks, such as the application of air quality detection, which was developed by University of California at Berkeley (UCB) Common Sense [1], the water pollution research, which was developed by IBM's Creek Watch [2], and the project to research the interaction between users and the environment according to location information, which was developed by California, University of Los Angeles (UCLA) PEIR [3]. Those are the research projects based on the crowd-sensing field. In addition, the health services project BikeNet [4], the combine of social perception and social networking project CenceMe [5] and the famous commercial map service company Waze (Waze) [6] are all crowd-sensing used in Social life.

In noise monitoring, Maisonneuve $\mathrm{N}$, Niessen $\mathrm{ME}$, et al proposed a city noise monitoring 
platform, NoiseTube [7], which uses smart phone to collect noise pollution data, and gets noise map after processing. To understand their daily noise exposure, people can check the noise maps on Google Earth. But the specific data collection strategies and data processing methods did not explain in this platform. In the literature [8], D'Hondt E, Stevens M, Jacobs A proved that it is reliable to obtain the noise information by using the crowd-sensing to get the noise map, compare with the method of standard comparison to obtain noise map. Liu and $\mathrm{Wu}$ constructed a noise-collection system, which is based on crowd-sensing [9-10]. This system corrected the error of microphone acquisition, and resumed the sparseness of data-collecting. But it did not cover the monitoring task distribution.

\section{The Constitute of Urban Noise complaint System Based on Mobile Crowd-Sensing}

This paper proposes that the urban noise complaint system, which is based on mobile crowd-sending is made up by smart phone and server (illustrated in Fig. 1).

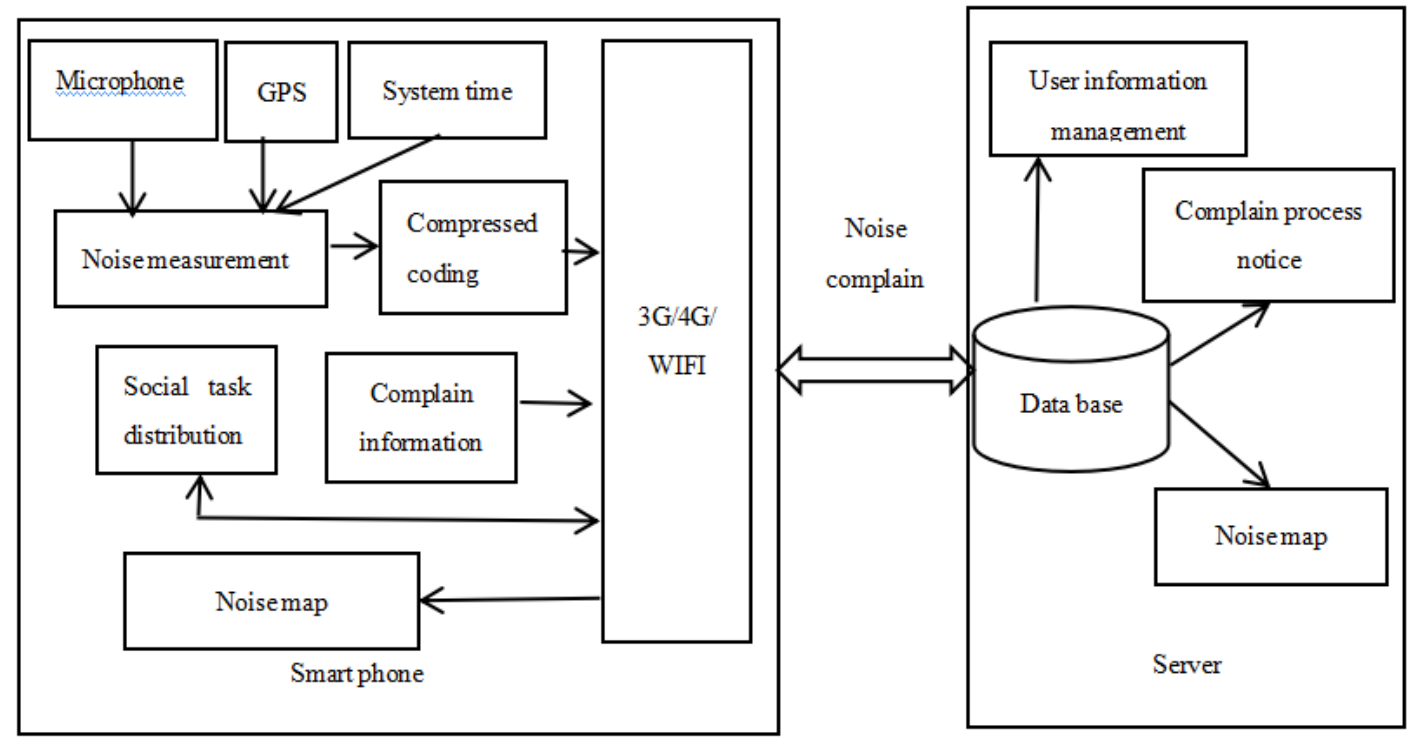

Figure 1. Finite The constitute of urban noise complaint system based on mobile crowd-sensing

For the smart phone terminal, the user can use the system time and GPS to obtain the time and location of noise-collection, and use the microphones to collect environmental noise. To the collected environmental noise, the system can use the noise measurement module to measure the noise volume and calculate the decibel of noise.

After obtaining the noise volume, the user can fill in the complaint target information, such as the name of the noise maker, the location of noise, the type of noise (such as building noise, life noise, traffic noise). After that, the complaint information and collected noise evidence are sent to the server through 3G / 4G / WIFI network.

Users can also check the noise map on the mobile. The noise map uses the Baidu map display and is divided into $50 \mathrm{~m} * 50 \mathrm{~m}$ grid. According to the noise level of each grid unit, the map will show different colors in corresponding block .

In order to reflect the noise pollution objectively, users can use the social task distribution module to distribute the noise monitoring complaint task to other users, and allow the other users to monitor the noise together to ensure the quality of noise monitoring.

On server terminal, the database keep the related information about the users and noise complaint. According to the user's complaint information, the noise map module draws the noise pollution situation on the Baidu map. According to the noise type by user's complaint, the complaint processing module sends the complaint information to the related department. 


\section{Noise Measurement}

For the noise complaint system designed in this paper, it is necessary to quantify the noise and report it as evidence. The A-weighted sound level can better reflect the subjective feeling to ears to the noise intensity and frequency. For a continuous and stable noise, it is a better evaluation method. However, the noise is undulating and discontinuous. It is more appropriate to measure the noise by equivalent continuous sound level A.

If the equivalent continuous sound level $\mathrm{A}$ is ${ }^{2}$, according to the definition of equivalent continuous sound level A, there is:

$$
L_{e q}=10 \lg \left\{\frac{1}{T} \int_{0}^{T} 10^{0.1 L_{A}} d_{t}\right\}
$$

Eq. 1: $L_{A}$ is the instantaneous value of the change A-weighted sound level, $\mathrm{T}$ is the total amount of some time.

In actual noise measurement, it can set the same sampling interval time for continuous sampling measurement, there is:

$$
L_{e q}=10 \lg \left(\frac{1}{N} \sum_{k=1}^{n} 10^{0.1 L_{A k}}\right)
$$

Eq. 2: $\mathrm{N}$ is the total number of measured sound levels, $L_{A k}$ is the k-th sampled sound level. Through the Eq. 2, it can measure the noise by smart phone.

\section{Social Task Distribution Mechanism Design}

In order to reflect the noise pollution objectively, it needs large number of users to involve in the process of monitoring complaints. This paper designs a Social task distribution mechanism that allows users to use Social networks to distribute the monitoring of complaints issued to other users. The other users can continue to forward the task, so that more and more users to participate in to ensure the monitoring quality. The Social task distribution mechanism is designed as follows:

If the i-th user's social activity is ${ }^{A_{i}}$, it uses to measure the i-th user can accept the number of friends to distribute tasks in period time $\mathrm{t}$. The greater value $A_{i}$ is, the more friends the $\mathrm{i}$-th user can know and the higher probability of task distribution to other users through social network. The calculation equations for $A_{i}$ is shown as Eq. 3:

$$
A_{i}=\frac{M_{i}}{N}
$$

In Eq. 3, $M_{i}$ is the $\mathrm{i}$-th user who has how many online friends in time $\mathrm{t}$, and $\mathrm{N}$ is the total number of online users in time $t$.

If the success rate of the $\mathrm{i}$-th user involved in the task distribution is $S_{i}$, the greater value $S_{i}$ is, the higher willingness of the i-th user to participate and distribute the task, and the higher probability that the task will continue to be distributed. $S_{i}$ is calculated as equations 4 :

$$
S_{i}=\frac{T_{s i}}{T_{i}}
$$

In Eq. $4, T_{s i}$ is the number of tasks that the i-th user has already distributed, and $T_{i}$ is the total number of the i-th user who participated and distributed tasks. 
If the probability of the i-th user who continue to distribute the task is $P_{i}$, obviously there is: $P_{i}=A_{i} \times S_{i}$

When a user involves a social task distribution, it needs to set monitoring time range and monitoring object. During this time, the user can distribute the monitoring complaint task to other users who has higher $P_{i}$ value. The other users can participate in the task, or can continue to do task distribution. When the number of users reaches the highest level required by system, the task can not be continued to forward. When the time specified in this task is over, the system allows users to start a new task distribution. The work flow is shown in Fig. 2:

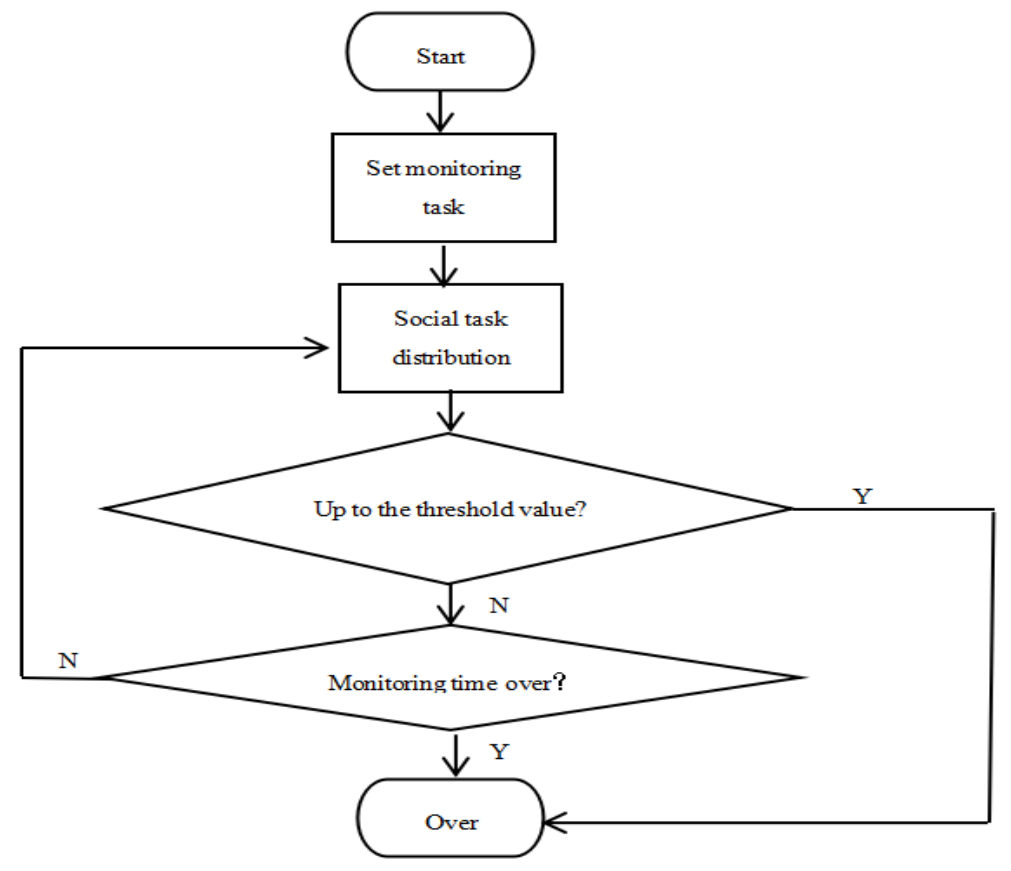

Figure 2. Finite The work flow for social task distribution

\section{Summary}

This paper designs and realizes the urban noise complaint system, which is based on Crowd-sensing. It solves the problem that evidence for city noise complaint is difficult to solidify and proposes the social task distribution mechanism, which makes more and more users willing to participate in the monitoring the noise complaint task. .

In future further studies, the user incentive mechanism will be introduced in, and it can continue to improve the quality of noise monitoring.

\section{Acknowledgements}

This work is supported by a project from Natural Science Foundation of Hainan Province (20156219) which presides by Hongli Wu.

\section{Reference}

[1] Dutta P, Aoki P M, Kumar N, et al. Common Sense: participatory urban sensing using a network of handheld air quality monitors[C]// International Conference on Embedded Networked Sensor Systems, SENSYS 2009, Berkeley, California, Usa, November. DBLP, 2009:349-350.

[2] Kim S, Robson C, Zimmerman $\mathrm{T}$, et al. Creek watch: pairing usefulness and usability for successful citizen science[C]// International Conference on Human Factors in Computing Systems, CHI 2011, Vancouver, Bc, Canada, May. DBLP, 2011:2125-2134. 
[3] Min M, Reddy S, Shilton K, et al. PEIR, the personal environmental impact report, as a platform for participatory sensing systems research[C]// International Conference on Mobile Systems, Applications, and Services. DBLP, 2009:55-68.

[4] Eisenman S B, Miluzzo E, Lane N D, et al. BikeNet: A mobile sensing system for cyclist experience mapping[J]. Acm Transactions on Sensor Networks, 2009, 6(1):6.

[5] Miluzzo E, Lane N D, Fodor K, et al. Sensing meets mobile social networks: the design, implementation and evaluation of the CenceMe application[C]// ACM Conference on Embedded Network Sensor Systems. ACM, 2008:337-350.

[6] Waze. Way to go! 2010.http://waze.com

[7] Maisonneuve N, Stevens M, Niessen M E, et al. NoiseTube: Measuring and mapping noise pollution with mobile phones[J]. Environmental Science \& Engineering, 2009, 2(6):215-228.

[8] D'Hondt E, Stevens M, An J. Participatory noise mapping works! An evaluation of participatory sensing as an alternative to standard techniques for environmental monitoring $[\mathrm{J}]$. Pervasive \& Mobile Computing, 2012, 9(5):681-694.

[9] LIU Lubin, ZHU Yanmin. Noise Collection and Presentation System Based on Crowd Sensing [J]. Computer Engineering, 2015, 41(10): 160-164.

[10] Wu W, Guo B, Yu Z. Crowd sensing based urban noise map and temporal-spatial feature analysis[J]. Journal of Computer-Aided Design \& Computer Graphics, 2014, 26(4):638-643. 